PROCEEDINGS OF THE

AMERICAN MATHEMATICAL SOCIETY

Volume 126, Number 7, July 1998, Pages 1909-1914

S 0002-9939(98)04543-2

\title{
DUALITY AND LOCAL GROUP COHOMOLOGY
}

\author{
P. R. HEWITT
}

(Communicated by Ronald M. Solomon)

\begin{abstract}
Let $G$ be a group, let $k$ be a field, and let $\mathcal{L}$ be a local system - an upwardly directed collection of subgroups whose union is $G$. In this paper we give a short, elementary proof of the following result: If either $A$ is a $k$ - $k G$-bimodule, or else $k$ is finite dimensional over its center, then $\operatorname{Ext}_{G}^{*}\left(A, B^{\vee}\right)=\varliminf_{L \in \mathcal{L}} \operatorname{Ext}_{L}^{*}\left(A, B^{\vee}\right)$. From this we deduce as easy corollaries some recent results of Meierfrankenfeld and Wehrfritz on the cohomology of a finitary module.
\end{abstract}

\section{MAIN THEOREM}

Let $G$ be a group, let $k$ be a field, and let $\mathcal{L}$ be a local system - an upwardly directed collection of subgroups whose union is $G$. Modules are $k$-spaces. If $X$ is a module, then $X^{\vee}$ denotes its dual, and $\langle\mid\rangle$ denotes their pairing. We will explore the relationship between the cohomology of $G, \operatorname{Ext}_{G}^{*}(A, B)$, with the local cohomology, $\operatorname{Ext}_{\mathcal{L}}^{*}(A, B)$. This latter is defined by taking the limit, with respect to restriction maps, of the cohomology groups $\operatorname{Ext}_{\mathcal{L}}^{*}(A, B)$, for $L$ in $\mathcal{L}$ :

$$
\operatorname{Ext}_{\mathcal{L}}^{*}(A, B):=\lim _{\longleftarrow} \operatorname{Ext}_{L}^{*}(A, B)
$$

It has long been known that, although homology can be determined locally, in general cohomology cannot. (In a later section we present two standard examples of this phenomenon.) Recently, Meierfrankenfeld [M] and Wehrfritz [W-2] have both published theorems concerning the local detection of finitary cohomology. It turns out that all of these results are subsumed by the following elementary theorem.

Theorem A. Let $A$ and $B$ be $G$-modules. If either $A$ is a $k$-kG-bimodule, or else $k$ is finite dimensional over its center, then localization

$$
\operatorname{Ext}_{G}^{*}\left(A, B^{\vee}\right) \rightarrow \operatorname{Ext}_{\mathcal{L}}^{*}\left(A, B^{\vee}\right)
$$

is an isomorphism.

Proof. Assume first that $A$ is a $k$ - $k G$-bimodule. We can combine the well-known fact that homology is determined locally, with an equally well-known duality between homology and cohomology:

$$
\operatorname{Ext}_{\mathcal{L}}^{*}\left(A, B^{\vee}\right)=\operatorname{Tor}_{*}^{\mathcal{L}}(A, B)^{\vee}=\operatorname{Tor}_{*}^{G}(A, B)^{\vee}=\operatorname{Ext}_{G}^{*}\left(A, B^{\vee}\right) .
$$

Continuity of homology with respect to direct limits follows from [S, Thm. 5.1.9] and $[\mathrm{S}$, Thm 4.1.7]. The duality appears in many guises in the literature. When

Received by the editors December 12, 1996.

1991 Mathematics Subject Classification. Primary $20 \mathrm{~J} 05$.

(C) 1998 American Mathematical Society 
$k$ is commutative, the form we use is a special case of [B, Prop. 2.8.5]. For the convenience of the reader who does not wish to wade into a vast sea of cohomology, we include the very simple proofs of these two facts in an appendix on local cohomology. These proofs work for skew fields as well as commutative ones.

To finish the proof, we now consider the case of a skew field that is finitedimensional over its center. If $\zeta$ is the center of $k$, and $\mu$ is a maximal commutative subfield of $k$, then

$$
\operatorname{Hom}_{k}\left(A, B^{\vee}\right) \otimes_{\zeta} \mu=\operatorname{Hom}_{\mu}\left(A, B^{\vee}\right) .
$$

Since field extension is flat, we obtain that

$$
\operatorname{Ext}_{G}^{*}\left(A, B^{\vee}\right) \otimes_{\zeta} \mu=\operatorname{Ext}_{\mathcal{L}}^{*}\left(A, B^{\vee}\right) \otimes_{\zeta} \mu,
$$

Hence $\operatorname{Ext}_{G}^{*}\left(A, B^{\vee}\right)=\operatorname{Ext}_{\mathcal{L}}^{*}\left(A, B^{\vee}\right)$, since field extension is faithfully flat.

\section{ApplicAtions}

Throughout this section we work under the hypotheses of Theorem A.

Corollary 1. Locally trivial cohomology classes are trivial in the double dual.

Proof. Extension of scalars from $B$ to $B^{\mathrm{W}}$ factors through localization:

$$
\begin{aligned}
\operatorname{Ext}_{G}^{*}(A, B) & \rightarrow \operatorname{Ext}_{G}^{*}\left(A, B^{\mathrm{W}}\right) \\
\downarrow & \| \\
\operatorname{Ext}_{\mathcal{L}}^{*}(A, B) & \rightarrow \operatorname{Ext}_{\mathcal{L}}^{*}\left(A, B^{\mathrm{W}}\right) .
\end{aligned}
$$

This following seems to be well-known, at least in the case where $A=k$.

Corollary 2. If $A$ is an arbitrary $G$-module and $B$ is a finite-dimensional $G$ module, then $\operatorname{Ext}_{G}^{*}(A, B)$ is determined locally.

For further applications, we can use Theorem A in conjunction with the long exact sequence associated to the extension $B \hookrightarrow B^{\mathrm{W}}$ :

$$
\cdots \rightarrow \operatorname{Ext}_{G}^{i-1}\left(A, B^{\mathrm{W}} / B\right) \rightarrow \operatorname{Ext}_{G}^{i}(A, B) \rightarrow \operatorname{Ext}_{G}^{i}\left(A, B^{\mathrm{W}}\right) \rightarrow \cdots
$$

Theorem B. If $A$ and $B$ are arbitrary $G$-modules, then the locally trivial portion of $\operatorname{Ext}_{G}^{i}(A, B)$ is covered by $\operatorname{Ext}_{G}^{i-1}\left(A, B^{\mathrm{W}} / B\right)$.

Proof. Apply Corollary 1 to $(\dagger)$.

We now turn to the cohomology of finitary modules. A transformation $x$ is finitary on $V$ in case $\operatorname{dim}_{k}[V, x]=\operatorname{dim}_{k} V / \mathrm{C}_{V}(x)<\infty$. The finitary transformations of $V$ form a normal subrgroup of $\mathrm{GL}(V)$, which is called the finitary group on $V$ and denoted $\mathrm{FGL}(V)$. Phillips' survey article $[\mathrm{Ph}]$ is an excellent introduction to finitary groups.

The definition of a finitary group is quantitative. The following qualitative characterization of finitary groups - due to Meierfrankenfeld - is the key to understanding their cohomology.

Proposition (Meierfrankenfeld).

$$
\operatorname{FGL}(V)=\mathrm{C}_{\mathrm{GL}(V)}\left(V^{\mathrm{W}} / V\right) \text {. }
$$


Proof. Let $x$ be a transformation of $V$. Apply Lemma 2 (below) to $x-1$ twice: between $V$ and $V^{\vee}$, and also between $V^{\vee}$ and $V^{\mathrm{W}}$. Next, use the fact that a space equals its double dual if and only if it is finite dimensional.

Corollary 3. Let $U$ be an arbitrary module, and $V$ a finitary module.

(a) If $U=[U, G]$, then localization $\operatorname{Ext}_{G}^{1}(U, V) \rightarrow \operatorname{Ext}_{\mathcal{L}}^{1}(U, V)$ is injective.

(b) If $\operatorname{Hom}(G, k)=0$, then localization $\mathrm{H}^{2}(G, V) \rightarrow \mathrm{H}^{2}(\mathcal{L}, V)$ is injective.

Proof. If $U=[U, G]$ then $\operatorname{Ext}_{G}^{0}\left(U, V^{\mathrm{W}} / V\right)=\operatorname{Hom}_{k G}\left(U, V^{\mathrm{W}} / V\right)=0$, whence we obtain part (a). If $\operatorname{Hom}(G, k)=0$ then $\operatorname{Ext}_{G}^{1}\left(k, V^{\mathrm{W}} / V\right)=\operatorname{Hom}\left(G, V^{\mathrm{W}} / V\right)=0$, which yields part (b).

Corollary 4 ([M, Thm. 1]). If $V$ is finitary and locally completely reducible, then $[V, G]$ is completely reducible.

Proof. Let $W<[V, G]$. We show that $W$ is a direct summand. Set $U=[V, G] / W$. Since $[V, G]=[V, G, G], \operatorname{Hom}_{G}\left(U, W^{\mathrm{W}} / W\right)=0$. Now apply Corollary $3(\mathrm{a})$.

The example in [W-1] shows that, without some hypothesis on the field, Corollary 4 would be false. (See also [ShW, Ex. 1.18].)

Corollary 5 ([M, Thm. 2]). If $\mathrm{H}^{1}(L, V)$ and $V / \mathrm{C}_{V}(L)$ are finite-dimensional for every $L$ in $\mathcal{L}$, then localization $\mathrm{H}^{2}(G, V) \rightarrow \mathrm{H}^{2}(\mathcal{L}, V)$ is injective.

Proof. If $V$ is finite dimensional, apply Corollary 2. If not, then $\mathrm{C}_{V}(L)$ is infinite dimensional, and so the exact sequence

$$
\cdots \rightarrow \mathrm{H}^{0}\left(L, V / \mathrm{C}_{V}(L)\right) \rightarrow \mathrm{H}^{1}\left(L, \mathrm{C}_{V}(L)\right) \rightarrow \mathrm{H}^{1}(L, V) \rightarrow \cdots
$$

tells us that $\mathrm{H}^{1}(L, k)=0$, since otherwise $\mathrm{H}^{1}(L, V)$ would also be infinite dimensional. Hence $\mathrm{H}^{1}(G, k)=0$. Now apply Corollary $3(\mathrm{~b})$.

Corollary 6 ([M, Thm. 3]). If $V \hookrightarrow W$ is a locally split extension of $V$ by a trivial $G$-module, then there is a canonical injection $W / \mathrm{C}_{W}(G) \hookrightarrow\left[V^{\vee}, G\right]^{\vee}$.

Proof. Let $U$ be the amalgamated sum of $W$ and $V^{\mathrm{W}}$ over $V$ :

$$
\begin{array}{lll}
W & \rightarrow & U \\
\uparrow & & \uparrow \\
V & \rightarrow & V^{W}
\end{array}
$$

By Corollary 1, the extension $V^{\mathrm{W}}<U$ is split - that is, $U=V^{\mathrm{W}}+\mathrm{C}_{U}(G)$. Thus,

$$
W / \mathrm{C}_{W}(G) \hookrightarrow U / \mathrm{C}_{U}(G)=V^{\vee} / \mathrm{C}_{V^{\vee}}(G)=\left[V^{\vee}, G\right]^{\vee} .
$$

We have now come full circle, since this paper is the direct result of generalizing [M, Thm. 3].

\section{TWO EXAMPLES}

In this section we explore two standard examples, which illustrate the discontinuous behavior of cohomology.

For the first example, let $k$ have odd characteristic, let $V$ be infinite-dimensional over $k$, endowed with a nondegenerate symplectic form, and let $G$ be the finitary symplectic group. The form gives an embedding of $V$ in $V^{\vee}$. The quotient $V^{\vee} / V$ is $G$-trivial (since $V^{\vee} / V$ is covered by $V^{\vee} / V$ ). The extension $V \hookrightarrow V^{\vee}$ is nonsplit 
since $\mathrm{C}_{V^{\vee}}(G)=0$. However, if we let $\mathcal{L}$ be the collection of symplectic groups of the finite-dimensional, nondegenerate subspaces of $V$, then we find that the extension is locally split. (See [CPS, Tbl. 4.5, pp. 186-187].) By Theorem A, any $G$-trivial extension of $V^{\vee}$ is split.

For the second example, let $\Omega$ be a set of some infinite cardinality $\aleph$, let $G$ be the finitary symmetric group on $\Omega$, let $k$ be of characteristic 2 , and let $V$ be the natural permutation module $k \Omega$. The Eckmann-Shapiro Lemma (see [B, Cor. 2.8.4]) tells us that $\operatorname{dim}_{k} \mathrm{H}^{1}\left(G, V^{\vee}\right)=1$ and $\operatorname{dim}_{k} \mathrm{H}^{2}\left(G, V^{\vee}\right)=2$. Now, as before, $V$ embeds in $V^{\vee}$, and the quotient is $G$-trivial. The long exact sequence contains the fragment

$$
\cdots \rightarrow \mathrm{H}^{1}\left(G, V^{\vee}\right) \rightarrow \mathrm{H}^{1}\left(G, V^{\vee} / V\right) \rightarrow \mathrm{H}^{2}(G, V) \rightarrow \mathrm{H}^{2}\left(G, V^{\vee}\right) \rightarrow \cdots .
$$

Thus $\operatorname{dim}_{k} \mathrm{H}^{2}(G, V)=\operatorname{dim}_{k} \mathrm{H}^{1}\left(G, V^{\vee} / V\right)=2^{\aleph}$. On the other hand, if we express $G$ as a union of finite symmetric groups, then we have that $\mathrm{H}^{2}(\mathcal{L}, V)=k$. To see this, suppose $\Omega=\Delta \sqcup \Gamma=\Delta^{\prime} \sqcup \Gamma^{\prime}$, with $\Delta$ and $\Delta^{\prime}$ finite, and with $\Gamma \supset \Gamma^{\prime}$. Set $F=\mathrm{C}_{G}(\Gamma), F^{\prime}=\mathrm{C}_{G}\left(\Gamma^{\prime}\right)$. Let $\gamma \in \Delta$. Now a cochain on a finite group takes values in a finite-dimensional subspace; hence its cohomology respects arbitrary direct-sum decompositions. Together with the Eckmann-Shapiro Lemma, this tells us that

$$
\begin{array}{r}
\mathrm{H}^{2}(F, V)=\mathrm{H}^{2}(F, k \Delta) \oplus \mathrm{H}^{2}(F, k \Gamma)=\mathrm{H}^{2}\left(F_{\gamma}, k\right) \oplus \bigoplus_{\gamma^{\prime} \in \Gamma} \mathrm{H}^{2}\left(F, k \gamma^{\prime}\right), \\
\left.\mathrm{H}^{2}\left(F^{\prime}, V\right)=\mathrm{H}^{2}\left(F^{\prime}, k \Delta^{\prime}\right)\right) \oplus \mathrm{H}^{2}\left(F^{\prime}, k \Gamma^{\prime}\right)=\mathrm{H}^{2}\left(F_{\gamma}^{\prime}, k\right) \oplus \bigoplus_{\gamma^{\prime} \in \Gamma^{\prime}} \mathrm{H}^{2}\left(F, k \gamma^{\prime}\right),
\end{array}
$$

where the subscripts denote point-stabilizers. The restriction map $\mathrm{H}^{2}\left(F^{\prime}, V\right) \rightarrow$ $\mathrm{H}^{2}(F, V)$ induces an isomorphism $\mathrm{H}^{2}\left(F_{\gamma}^{\prime}, k\right) \rightarrow \mathrm{H}^{2}\left(F_{\gamma}, k\right)$ (see [AM, ch. VII]) and a canonical embedding on the remaining factors. Thus, taking successively smaller $\Gamma^{\prime}$, we see that none of the classes in $\mathrm{H}^{2}(F, V)$ that take values in $k \Gamma$ survive in the limit.

Although very little of the degree-2 cohomology of the symmetric group is detected locally, all of the degree- 2 cohomology for the alternating subgroup is detected locally, by Corollary 3. Moreover, all of the degree-2 cohomology for the symmetric group is detected locally when we replace $k$ by a field of any other characteristic.

\section{LOCAL COHOMOLOGY}

In this section we provide simple, direct proofs of the facts used in the proof of Theorem A. These facts are well-known when the base field is commutative, but much less so when the field is skew.

As before, let $G$ be a group, let $k$ be a field, and let $\mathcal{L}$ be a local system in $G$.

We begin with a discussion of local group cohomology, from the familiar point of view of cochains and coboundary. Just as with the definition of local cohomology, above, we can use the restriction maps to define analogous limits, $\mathrm{C}^{*}(\mathcal{L}, V)$, $\mathrm{Z}^{*}(\mathcal{L}, V)$, and $\mathrm{B}^{*}(\mathcal{L}, V)$, for cochains, cocycles, and coboundaries, respectively. What we find is that there are canonical isomorphisms $\mathrm{C}^{*}(\mathcal{L}, V) \cong \mathrm{C}^{*}(G, V)$ and $\mathrm{Z}^{*}(\mathcal{L}, V) \cong \mathrm{Z}^{*}(G, V)$. Indeed, the localization $\mathrm{C}^{*}(G, V) \rightarrow \mathrm{C}^{*}(\mathcal{L}, V)$ is defined by restriction to the local subgroups, $\phi \mapsto\left\{\left.\phi\right|_{L}\right\}$. In the reverse direction, the map $\mathrm{C}^{*}(\mathcal{L}, V) \rightarrow \mathrm{C}^{*}(G, V)$ splices an $\mathcal{L}$-sequence of cochains $\left\{\phi_{L}\right\}$ into a cochain on $G: g_{1}, \ldots, g_{n} \mapsto \phi_{L}\left(g_{1}, \ldots, g_{n}\right)$, where $L$ is any member of $\mathcal{L}$ that contains all the 
$g_{i}$. This is well-defined, since in the inverse limit $\mathrm{C}^{*}(\mathcal{L}, V),\left.\phi_{L}\right|_{L^{\prime}}=\phi_{L^{\prime}}$, whenever $L \supset L^{\prime}$.

These maps respect coboundary, and so they induce isomorphisms for the cocycle groups. However, splicing an $\mathcal{L}$-sequence of coboundaries need not yield a coboundary in $G$. In general we obtain only an embedding $\mathrm{B}^{*}(G, V) \hookrightarrow \mathrm{B}^{*}(\mathcal{L}, V)$.

Now consider the localization $\mathrm{H}^{*}(G, V) \rightarrow \mathrm{H}^{*}(\mathcal{L}, V)$. An element of $\mathrm{H}^{i}(\mathcal{L}, V)$ is an inverse system of affine flats $\left\{\phi_{L}+\mathrm{B}^{i}(L, V)\right\}$, where $\left.\phi_{L}\right|_{L^{\prime}} \equiv \phi_{L^{\prime}} \bmod \mathrm{B}^{i}\left(L^{\prime}, V\right)$ whenever $L \supset L^{\prime}$. The image of $\mathrm{H}^{i}(G, V)$ consists of those inverse systems whose inverse limit is nonempty. Similarly, an element in the kernel of the map $\mathrm{H}^{i+1}(G, V) \rightarrow$ $\mathrm{H}^{i+1}(\mathcal{L}, V)$ is an inverse system of affine flats $\left\{\psi_{L}+\mathrm{Z}^{i}(L, V)\right\}$, where $\left.\delta \psi_{L}\right|_{L^{\prime}}=\delta \psi_{L^{\prime}}$ whenever $L \supset L^{\prime}$ — viewed modulo the collection of those inverse systems that have a nonempty limit.

The simple 'compactness' proof of [M, Thm. 2] applies in arbitrary cohomological degree, to give the following generalization.

Proposition. Let $i$ be a positive integer.

(a) If $\operatorname{dim}_{k} \mathrm{~B}^{i}(L, V)<\infty$ for all $L$ in $\mathcal{L}$, then localization $\mathrm{H}^{i}(G, V) \rightarrow \mathrm{H}^{i}(\mathcal{L}, V)$ is surjective in degree $i$.

(b) If moreover $\operatorname{dim}_{k} \mathrm{Z}^{i}(L, V)<\infty$ for all $L$ in $\mathcal{L}$, then, additionally, localization $\mathrm{H}^{i+1}(G, V) \rightarrow \mathrm{H}^{i+1}(\mathcal{L}, V)$ is injective in degree $i+1$.

We will write $\operatorname{Ext}_{G}^{*}(A, B)$ instead of $\mathrm{H}^{*}\left(G, \operatorname{Hom}_{k}(A, B)\right)$, and $\operatorname{Tor}_{*}^{G}(A, B)$ instead of $\mathrm{H}_{*}\left(G, A \otimes_{k} B\right)$. In addition to local cohomology, we could also define local homology: $\operatorname{Tor}_{*}^{\mathcal{L}}(A, B)=\underline{\mathrm{lim}} \operatorname{Tor}_{*}^{L}(A, B)$. However, this yields nothing new.

Lemma 1. If $A$ is a right $k G$-module and $B$ is a left $k G$-module, then

$$
\operatorname{Tor}_{*}^{\mathcal{L}}(A, B)=\operatorname{Tor}_{*}^{G}(A, B) .
$$

Proof. First of all, note that if $P_{*}=\mathrm{C}_{*}(G, \mathbb{Z})$, and $X$ is any $G$-module, then $\underline{\varliminf} P_{*} \otimes_{L} X=P_{*} \otimes_{G} X$. To see this, observe that $P_{*} \otimes_{G} X=\left(P_{*} \otimes X\right) /\left[P_{*} \otimes X, G\right]$ and $\left[P_{*} \otimes X, G\right]=\sum_{L}\left[P_{*} \otimes X, L\right]$.

Since the maps $P_{*} \otimes_{L} X \rightarrow P_{*} \otimes_{G} X$ are surjective, we obtain that the boundaries of the limit are limits of boundaries. Now if an element maps to 0 in a direct limit then it maps to 0 at some finite stage. This tells us that the cycles of the limit are limits of cycles.

Lemma 2. If $X$ is a $k$-space and $f: X \rightarrow X$ is a linear endomorphism, then

$$
\begin{array}{ll}
\operatorname{ann}_{X}\left(\operatorname{im}\left(f^{\vee}\right)\right)=\operatorname{ker}(f), & \operatorname{ann}_{X \vee}(\operatorname{im}(f))=\operatorname{ker}\left(f^{\vee}\right), \\
\operatorname{ann}_{X}\left(\operatorname{ker}\left(f^{\vee}\right)\right)=\operatorname{im}(f), & \operatorname{ann}_{X}(\operatorname{ker}(f))=\operatorname{im}\left(f^{\vee}\right) .
\end{array}
$$

Proof. The first two equalities follow from the identity $\langle f(x) \mid \lambda\rangle=\left\langle x \mid f^{\vee}(\lambda)\right\rangle$. The third follows from the first, since any subspace of $X$ is the annihilator of its annihilator. The last follows from composition of the canonical isomorphisms $\operatorname{ann}_{X} \vee(\operatorname{ker}(f))=\operatorname{im}(f)^{\vee}=\operatorname{im}\left(f^{\vee}\right)$.

Lemma 3. If $A$ is a $k$-kG-bimodule and $B$ is an arbitrary left $k G$-module, then

$$
\operatorname{Ext}_{G}^{*}\left(A, B^{\vee}\right)=\operatorname{Tor}_{*}^{G}(A, B)^{\vee} .
$$

Proof. Let $P_{*}=\mathrm{C}_{*}(G, \mathbb{Z})$, and note the canonical isomorphism

$$
\operatorname{Hom}\left(P_{*}, \operatorname{Hom}_{k}\left(A, B^{\vee}\right)\right)=\left(P_{*} \otimes A \otimes_{k} B\right)^{\vee} .
$$


This isomorphism can be described as follows: a functional $\lambda$ in $\left(P_{*} \otimes A \otimes_{k} B\right)^{\vee}$ is paired to an additive map $f: P_{*} \rightarrow \operatorname{Hom}_{k}\left(A, B^{\vee}\right)$ when they satisfy the relation

$$
\langle b \mid f(c)(a)\rangle=\langle c \otimes a \otimes b \mid \lambda\rangle .
$$

Now if we take fixed points for $G$ in this isomorphism, we obtain that

$$
\operatorname{Hom}_{G}\left(P_{*}, \operatorname{Hom}_{k}\left(A, B^{\vee}\right)\right)=\left(P_{*} \otimes_{G}\left(A \otimes_{k} B\right)\right)^{\vee} .
$$

To finish, apply Lemma 2 to the boundary of $P_{*} \otimes_{G}\left(A \otimes_{k} B\right)$.

\section{ACKNOWLEDGMENT}

It is a pleasure to record my thanks to Ulrich Meierfrankenfeld, for his many insights, suggestions, and patient explanations; and also to the Math Department at Michigan State University, for their generous hospitality. In addition, I wish to thank the referee for many helpful suggestions.

\section{REFERENCES}

[AM] A Adem, J Milgram, Cohomology of Finite Groups, Springer-Verlag, 1994. MR 96f:20082

[B] D Benson, Representations and Cohomology I, Cambridge University Press, 1991. MR 92m:20005

[CPS] E Cline, B Parshall, L Scott, Cohomology of finite groups of Lie type, I, Publ Math IHES 45 (1975), 169-191. MR 53:3134

[M] U Meierfrankenfeld, A note on the cohomology of finitary modules, Proc AMS (to appear).

$[\mathrm{Ph}] \quad \mathrm{R}$ Phillips, Finitary linear groups: a survey, Finite and Locally Finite Groups, Kluwer Academic Publishers, 1995, pp. 111-146. MR 96m:20080

[ShW] M Shirvani, B Wehrfritz, Skew Linear Groups, Cambridge University Press, 1986. MR 89h:20001

[S] E Spanier, Algebraic Topology, McGraw-Hill, 1966. MR 35:1007

[W-1] B Wehrfritz, Complete reducibility in skew linear groups, Jour LMS 28 (1983), 301-309. MR 85g:20020

[W-2] B Wehrfritz, The complete reducibility of locally completely reducible finitary linear groups, Bull LMS 29 (1997), 173-176. CMP 97:06

Department of Mathematics, University of Toledo, Toledo, Ohio 43606

URL: http://www.math.utoledo.edu/ ${ }^{\text {phewitt/ }}$ 